\title{
Grain Growth, Porosity, and Hardness Changes in Sintered and Annealed Binder- jet 3D Printed Ni-Mn-Ga Magnetic Shape Memory Alloys
}

Aaron Acierno ${ }^{1}$, Jakub Toman ${ }^{2}$, Katerina Kimes ${ }^{2}$, Amir Mostafaei ${ }^{3}$, Mirko Boin ${ }^{4}$, Robert Wimpory ${ }^{4}$ and Markus Chmielus ${ }^{2}$

${ }^{1}$ University of Pittsburgh, Glenshaw, Pennsylvania, United States, ${ }^{2}$ University of Pittsburgh, Pittsburgh, Pennsylvania, United States, ${ }^{3}$ Illinois Institute of Technology, Chicago, Illinois, United States, ${ }^{4}$ Helmholtz-Zentrum Berlin für Materialien und Energie, Berlin, Berlin, Germany

Stoichiometric Ni2MnGa-derivative Heusler alloys are classified as magnetic shape memory alloys (MSMAs) and demonstrate a large mechanical response under an externally applied magnetic field [1,2]. Large lattice deformation is the result of magnetic-field induced strain (MFIS), and occurs due to twin boundary motion of martensitic twins in the low-temperature ferromagnetic martensitic phase from its large magnetocrystalline anisotropy [2,3]. Lázpita et al. showed that fine-grained and randomly textured Ni-Mn-Ga samples exhibit hardly any MFIS under an external magnetic field due to internal constraints on twin boundary motion [4]. Recently, there has been interest in manufacturing Ni-Mn-Ga systems containing a porous network over a completely dense polycrystalline sample in order to enhance the MFIS [5]. According to Müllner et al., the MFIS is highly diminished in solid polycrystalline Ni-Mn-Ga as the grain boundaries inhibit the movement of twin boundaries. Oppositely, porous Ni-Mn-Ga materials allow the martensitic phase twins move with less resistance, decreasing the activation stress required for twin movement. [6-9]. In this study, neutron diffraction was performed on binder-jet printed Ni-Mn-Ga alloys which were sintered at different temperatures. The data from these measurements show a large difference in Bragg diffraction peaks due to grain size difference sintered at $1080{ }^{\circ} \mathrm{C}$ and $1090{ }^{\circ} \mathrm{C}$ as revealed by the images obtained by the area detector and grain boundaries revealed during etching. Also, the decrease in porosity with increasing sintering temperature resulted in an increase in hardness.

Sample preparation for the samples used in this experiment is provided in detail by Ref. [10]. Neutron diffraction was performed at the Helmholtz Centre Berlin for Materials and Energy (HZB) on beamline E3 $(1.47318 \pm 0.00056 \AA)$ on cut Ni-Mn-Ga samples. The beamline utilized an Si (400) plane monochromator and a PSD $30 \times 30 \mathrm{~cm}^{2}$ detector. Diffraction intensity was recorded at $2 \theta$ values of $42^{\circ}$, $53^{\circ}, 64^{\circ}$ and $75^{\circ}$ while $\omega$ was rotated in increments of $2^{\circ}$ from $-90^{\circ}$ to $0^{\circ}$ with the $\chi$ constant at $0^{\circ}$. Neutron diffraction area detector images were extracted with CARESSplorer and curves were generated using StressTex, and OriginPro fit Gaussian peaks to the diffraction curves. 2D diffraction images were processed using ImageJ image analysis software and MATLAB [11,12]. Nanoindentation was performed on mounted samples using a Hysitron TI 950 Triboindenter with a Berkovich indentation tip. Samples were tested in a trapezoidal loading function with the following steps: linear loading from $0 \mu \mathrm{N}$ to 2000 $\mu \mathrm{N}$ at a rate of $1000 \mu \mathrm{N} / \mathrm{sec}$, a constant applied load of $2000 \mu \mathrm{N}$ for $5 \mathrm{sec}$, and a linear unloading from $2000 \mu \mathrm{N}$ to $0 \mu \mathrm{N}$ at a rate of $-1000 \mu \mathrm{N} / \mathrm{sec}$. Tests were performed at various distances from pores on the sample interface, and the reduced Elastic modulus, $E_{r}$, and hardness were recorded in GPa. Loaddisplacement curves were collected and analyzed for all four of the samples in order to consider martensitic twin boundary motion.

Diffraction rings taken in the $\sim 42^{\circ}$ regime show three particular diffraction representing the (220) plane family, and are peaks characteristic of $14 \mathrm{M}$ martensite. The lattice parameters were calculated for 
diffraction tests performed on all of the samples using the (400) and (220) plane families. The c/a ratio is similar for all samples between 0.8942 and 0.8965 correspond to a $14 \mathrm{M}$ pseudo-orthorhombic martensitic structure [13]. Neutron data was processed image-by-image using a program written in MATLAB to help identify distinct clusters of high-neutron count pixels. Figure 1 shows an example of integrated (top) and one single (bottom) detector image following processing for the (220) plane family of 14M Ni-Mn-Ga martensite. It is seen that the $1090{ }^{\circ} \mathrm{C}$ sintered samples have much more easily distinguishable diffraction clusters compared to the $1080{ }^{\circ} \mathrm{C}$ sintered samples.

Nanoindentation (results summarized in the table below) was performed using a trapezoidal loading curve to a maximum force of $2000 \mu \mathrm{N}$ on the surface of all samples at various distances from the pores and the reduced elastic modulus and hardness were recorded in GPa. In the table below, 'heat-treated' is shortened to 'HT'.

Hardness

Units

Sample

1080 non-HT

3.00

0.24

Std. Dev.

Average

[GPa]

[GPa]

[GPa]

Reduced Elastic Modulus

$1080 \mathrm{HT}$

3.36

0.37

88.3

13.7

1090 non-HT

3.47

0.24

104.9

12.2 
Between the four samples, the $1080{ }^{\circ} \mathrm{C}$ non-HT sample has the lowest hardness, and the samples sintered at $1090{ }^{\circ} \mathrm{C}$ have a larger hardness and reduced elastic modulus. Force-displacement curves taken on the samples suggest that there exist critical applied force thresholds where a brief increase in the deformation rate is observed. For each sample, during the loading portion of the force-displacement curve, sudden discontinuous jumps, so-called pop-in events, were recorded and suggested to signify the threshold movement forces required for martensitic phase boundary movement. In figure 2, representative forcedisplacement curves for the $1080{ }^{\circ} \mathrm{C}$ (left) and $1090{ }^{\circ} \mathrm{C}$ sintered and heat treated sample (right) are provided. It can be seen that low-load nanoindentation testing can be used in assessing the twin boundary movement behavior of porous Ni-Mn-Ga matrices, as the primary mode of plastic deformation can be limited to that of local glissile dislocation movement of the 14M martensitic phase. As noted by Aaltio et al. [14], these discontinuities are representative twin boundary movements in this experiment which has overcome the necessary external force threshold to travel about the sample [15]. Behavior of the forcedisplacement loading curve observed in our samples contain values and shape similar to that observed by Aaltio et al., indicating similar micro-mechanical properties of binder-jet 3D-printed and sintered Ni-MnGa.

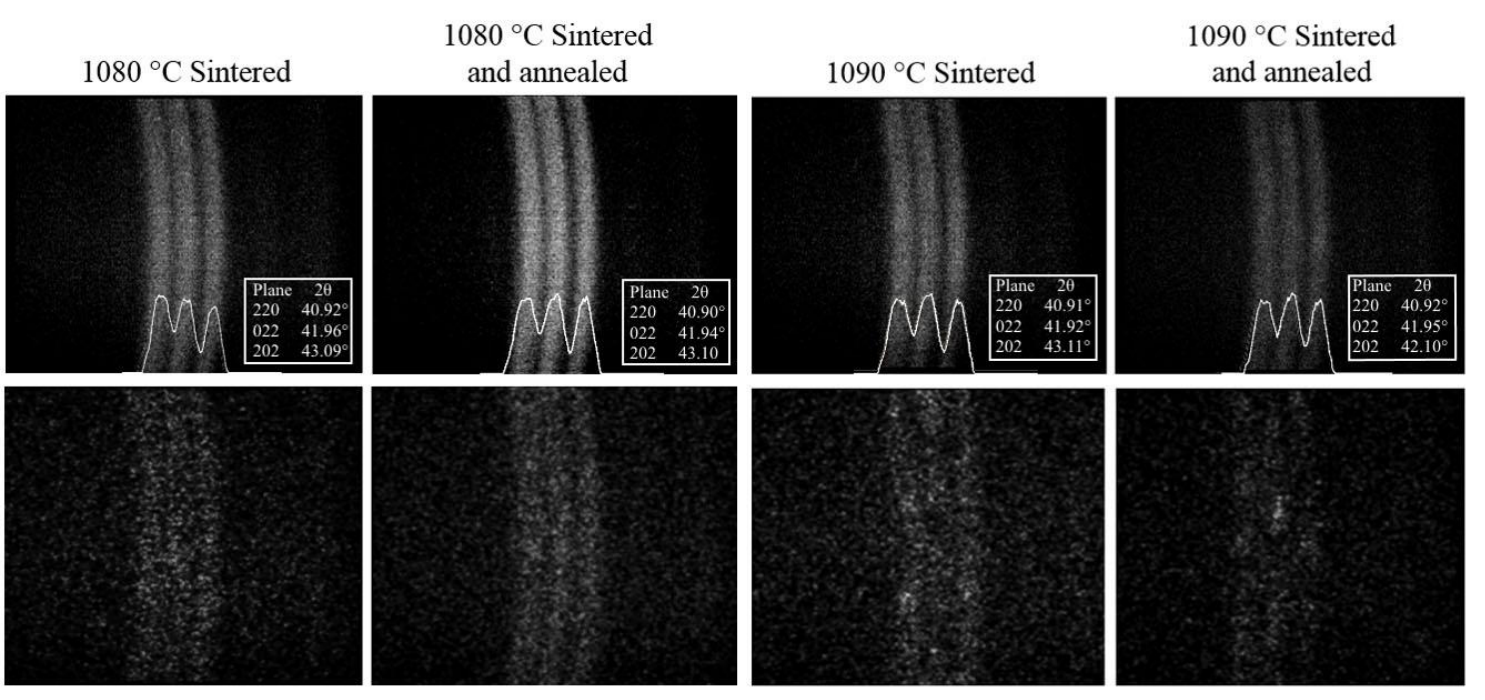

Figure 1. Comparison images of processed area detector diffraction images for each of the four samples. Images are taken of the (220) plane family of 14M Ni-Mn-Ga martensite. Insets of each diffraction image display the exact location and extracted summated diffraction curve for each band. The diffraction angles were used to determine lattice parameters for each sample. 

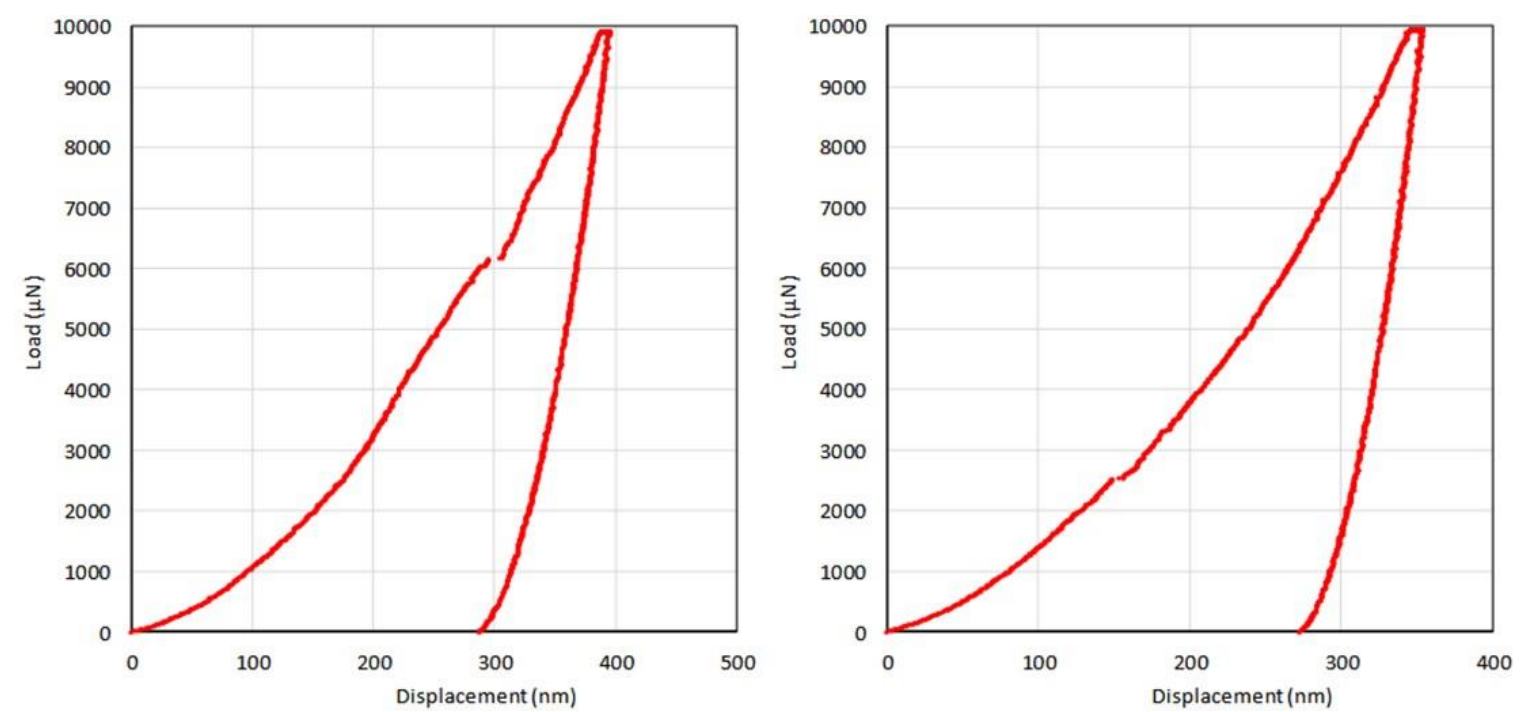

Figure 2. Force-displacement curves extracted from nanoindentation tests on the $1080{ }^{\circ} \mathrm{C}$ (left) and 1090 ${ }^{\circ} \mathrm{C}$ (right) sintered and heat treated samples. It is noted that a discontinuous jump in the displacement and various inflection points are observable along the loading portion of the force-displacement curve.

References

[1] K. Ullakko, J. K. Huang, C. Kantner, R. C. O’Handley, and V. V. Kokorin, Appl. Phys. Lett. 69 (1996) 1966-1968.

[2] K. Ullakko, J. Mater. Eng. Perform. 5 (1996) 405-409.

[3] C.P. Henry, D. Bono, J. Feuchtwanger, S.M. Allen, R.C. O’Handley. J. Appl. Phys. 91 (2002) 7810.

[4] P. Lázpita, G. Rojo, J. Gutiérrez, J.M. Barandiaran, R.C. O’Handley, Sensor Letters. 5 (2007) 65-68.

[5] M. Caputo, C.V. Solomon, P. Nguyen, A.E. Berkowitz. Proc. Microsc. Microanal. (2016) 1770-1771.

[6] P. Müllner, V.A. Chernenko, G. Kostorz, J. Appl. Phys. 95 (2004) 1531.

[7] C. Witherspoon, P. Zheng, M. Chmielus, D.C. Dunand, P. Müllner, Acta Mater. 92 (2015) 64-71.

[8] Y. Boonyongmaneerat, M. Chmielus, D.C. Dunand, P. Müllner, Phys. Rev. Lett. 99 (2007) 247201.

[9] M. Chmielus, X.X. Zhang, C. Witherspoon, D.C. Dunand, P. Müllner, Nature Mater. 8 (2009) 863866.

[10] A. Mostafaei, P.R. De Vecchis, E.L. Stevens, M. Chmielus, Acta Mater. 154 (2018) 355-364.

[11] C.T. Rueden, J. Schindelin, M.C. Hiner, B.E. DeZonia, A.E. Walter, E.T. Arena, K.W. Eliceiri, BMC Bioinformatics. 18 (2017) 529.

[12] J. Schindelin, I Arganda-Carreras, E. Frise, V. Kaynig, M. Longair, T. Pietzsch, S. Prebisch, C. Rueden, S. Saalfeld, B. Schmid, J.Y Tinevez, D.J. White, V. Hartenstein, K. Eliceiri, P. Tomancak, A. Cardona, Nat. Methods. 9 (2012) 676-682.

[13] K. Lu, W.T. Reynolds, Powder Technol. 187 (2008) 11-18.

[14] I. Aaltio, X.W. Liu, M. Valden, K. Lahtonen, O. Söderberg, Y. Ge, S-P. Hannula, Jour. Of Alloys and Compounds. 577 (2013) S367-S371.

[15] P.H. Davis, C.M. Efaw, L.K. Patten, C. Hollar, C.S. Watson, W.B. Knowlton, P. Müllner, Jour. Appl. Phys. 123 (2018) 215102. 